\title{
ESTUDIO DEL ROL DE LOS PADRES DE FAMILIA EN LA VIDA EMOCIONAL DE LOS HIJOS
}

\author{
Kennya Guzmán Huayamave ${ }^{1 \mathrm{a}}$, Brenda Bastidas Benavides² y Mercedes Mendoza \\ Sangacha ${ }^{3}$ \\ Universidad Laica Vicente Rocafuerte de Guayaquil ${ }^{123}$ \\ Orcid ID: https://orcid.org/0000-0003-1204-27291 \\ Orcid ID: https://orcid.org/0000-0002-0581-202x² \\ Orcid ID: https://orcid.org/0000-0003-3689-2613³
}

Recibido: 03 de junio 2018

Aceptado: 06 de enero 2019

\section{Resumen}

El presente estudio, analiza la importancia del rol de la familia como principal agente socializador que debe favorecer el desarrollo emocional del niño y asegure una identidad personal y social. Por lo que, enseñar en estos espacios, es una tarea compleja que genera ansiedad para que las familias logren cumplir con el rol establecido por la sociedad y sobre todo con la vocación de ser padres. El objetivo de la investigación, es determinar cómo influye el rol de los padres en el comportamiento emocional de los niños de 3 años. La metodología utilizada tiene un enfoque mixto. Para la fundamentación, aborda esencias teóricas de las variables y utiliza técnicas de observación y encuesta. A partir de los resultados, los padres de familia deben favorecer el aprendizaje de las competencias emocionales ya que dejan una huella invaluable para su crecimiento integral.

Palabras clave: Rol de los padres de familia, convivencia, emociones

\section{STUDY OF THE ROLE OF PARENTS IN THE EMOTIONAL LIFE OF THEIR CHILDREN}

\begin{abstract}
The present study analyzes the importance of the role of the family as the main socializing agent that should favor the emotional development of the child and ensure a personal and social identity. Therefore, teaching in these spaces is a complex task that generates anxiety for families to achieve the role established by society and above all with the vocation of being parents. The objective of the research study is to determine how the role of parents influences the emotional behavior of 3-year-old children. The methodology used has a mixed approach.
\end{abstract}

${ }^{\text {a }}$ Correspondencia al autor

E-mail: kguzmanh@ulvr.edu.ec 
At a foundational level, it addresses the theoretical essence of the variables and uses observation and survey techniques. Based on the results, parents should favor the learning of emotional competences since they leave an invaluable mark for their holistic growth.

Keywords: Role of parents, coexistence, emotions

\section{Introducción}

La sociedad de hoy en día transmite el reflejo de un estado donde los modelos de subsistencia han llevado a los padres a dedicar más tiempo al trabajo, y confiar a terceras personas la responsabilidad que es indelegable de los padres de familia para cubrir las necesidades que se presenten en sus hijos. Soler, Aparicio, Díaz, y Rodríguez (2016, p. 37) refieren que: "Desde la infancia nuestro bienestar viene determinado por las relaciones positivas con nuestros padres y familiares”. De esto se desprende que, el rol que desempeñan los padres es gravitante en el quehacer humano en todas las sociedades (Balabarca, 2018).

Trabajar en el desarrollo emocional siempre es un tema que enriquece a los padres de familia como testimonios de vida y modelos que marcan una identidad personal y social en los hijos. Esta investigación plantea la preocupación de los padres con respecto al tiempo que comparten con los hijos y la responsabilidad establecida para modelar las competencias emocionales, está orientada a reflexionar en el rol de los padres y el fortalecimiento del desarrollo emocional de los hijos.

Melgosa (2017, p.85) resalta en relación a las emociones que: "Las relaciones funcionan de forma cíclica: Cuando alguien es simpático y cariñoso, quienes lo observan, reaccionan favorablemente". Es decir, que todas emociones que el niño recibe son útiles para la supervivencia y el adulto está llamado a enseñarle desde temprana a edad a reconocer las emociones y las consecuencias que acarrea para la persona y los demás, cuando ellas son negativas.

Márquez y Gaeta (2017, p. 227) señalan que: “(...) los padres constituyen un factor importante el desarrollo de competencias emocionales, y puede hacerlo actuando directamente sobre sus hijos o de manera indirecta". Los investigadores reafirman la necesidad de una educación emocionalmente sana y, la mayor atención en los primeros años de vida del niño en el contexto familiar, con la debida regulación emocional y trabajo en la elaboración de respuestas adecuadas con el medio. 
Mancero (2013, p. 9) refiere que: “(...) el desarrollo de sociedades enteras se define por la calidad de su desarrollo infantil, puesto que el cerebro de los niños termina de desarrollarse en los primeros 3 años de vida”. Es en la infancia donde se aprende más que en cualquier otra etapa de la vida, donde los niños son sensibles a repetir las acciones que observan a lo largo de su niñez, al momento de crecer los niños imitarán las acciones que observaron y experimentaron en su entorno familiar, esencialmente de sus padres, representantes o personas que los cuidaron. Ibarrola $(2014$, p. 7) manifiesta que al niño: “Conviene transmitirle empatía con sus sentimientos, y a partir de ahí enseñarle a controlar sus emociones, para poder desarrollar un mundo emocional más equilibrado “. Al respecto, la relación afectiva debe ser atendida por sus padres y tener una comunicación empática le permite expresar y controlar sus emociones.

La problemática a resolver es: ¿Cuál debe de ser el rol de los padres de familia en el comportamiento emocional de los niños de 3 años?; ¿Para qué se debe desarrollar la comunicación "padres-hijos” desde temprana edad?; ¿Qué importancia tiene la influencia del comportamiento de los padres en el desarrollo emocional de los niños?

\section{Participación emocional de los padres en la educación infantil}

UNICEF (2012, p. 11) sostiene que: "El niño tiene, desde su nacimiento, la capacidad fundamental de relacionarse socialmente. Pero podrá desarrollarla, siempre y cuando haya alguien, el cuidador primario, disponible para establecer esta relación social". Se desprende que la familia es un vehículo de valores y conocimientos que son fundamentales para el bienestar emocional y define elementos para el resto de su vida. En correspondencia con lo expresado, Suárez y Vélez (2018, p.176) asumen:

De igual manera la familia cumple funciones como la preparación para ocupar roles sociales, control de impulsos, valores, desarrollo de fuentes de significado como, por ejemplo, la selección de objetivos de desarrollo personal, siendo esta socialización la que permite que los niños se conviertan en miembros proactivos de la sociedad.

Por estas razones, la familia es una red de apoyo para que estén construidas en valores y principios que fortalezcan su formación integral; sin embargo, en la actualidad la mayoría de los trabajos solicitan que sus empleados estén en constante actualización de conocimientos lo cual genera reducción del tiempo destinado a las actividades juntos a sus hijos, ya sean 
estás académicas, recreativas y familiares. En el caso de Ecuador, se procura brindar la atención a los vínculos afectivos en los primeros años de vida y que se asientan bases en el desarrollo emocional. Matamoros (2015, p.2) recalca:

En la sociedad actual, no existe una concienciación del impacto que tiene la familia en el desarrollo integral de los niños, especialmente durante los primeros años de vida. El rol de la familia y la interacción permanente entre sus miembros son determinantes en el desarrollo de valores del niño, pues se espera que el ambiente familiar sea propicio para poder potenciar este desarrollo.

De acuerdo a lo expresado, los padres de familia más allá de lo laboral, deben prestar atención al comportamiento emocional de sus hijos y se hace necesario que se organicen los tiempos para que estén vinculados con los acontecimientos en los contextos socializadores externos como son la escuela y los amigos; sin embargo, nadie puede responder a las exigencias del conocimiento, si antes no atiende la grandeza de su humanidad.

Con todo lo expresado, de nada sirve que a un niño se lo eduque en sus emociones si en la casa existen rutina que no proporcionan coherencia en sus discursos para una mayor compresión el padre debe ser un modelo emocional y para ello es necesario conocer la realidad objetiva que vive la familia:

\section{Tabla 1}

Una mirada holística a la clasificación de las familias

\section{Clasificación de las familias según su composición \\ Clasificación de acuerdo al tipo de convivencia}

\author{
Nuclear: Está conformada por papá, \\ mamá e hijos.
}

Extendida: Se encuentra uno de los padres y se suman con otros familiares: abuelos, tíos, otros.

Monoparental: Por divorcio, muerte, separación y está un padre o madre con sus hijos.

Homoparental: La conforman las parejas del mismo sexo y los hijos son adoptados, inseminación o de una

\begin{abstract}
Padres autoritarios. Tiene un excesivo
control en sus hijos y al no ser afectuosos con sus hijos, descuidan las necesidades emocionales.
\end{abstract}

Sobreprotector: Cuidan sus hijos al extremo y eso le impide que crezca y desarrolla con más facilidad miedos y fobias.

Permisiva: Proporcionan tanta libertad a los hijos que siempre intentan razonar las normas para establecer acuerdos con ellos. 
relación anterior hay un hijo de forma natural.

Ensamblada: La conforman hijos de la actual pareja y de uniones anteriores.

De hecho: Esta expresión indica familias de unión libre.
Por todo esto tienen la autoestima alta, son autónomos; sin embargo, al no haber tenido límites desde pequeños, les cuesta enfrentarse a problemas y relacionarse con los demás. Inestable: Afecta al niño a lo largo de su vida.

Estable: Son familias comprometidas y contribuyen a la seguridad social.

\section{Fuente: Elaborado por las autoras}

De todo esto se desprende que el ámbito familiar que el estilo parental se relaciona con la competencia emocional y social que promueven en los hijos. Ramírez, Ferrando y Sáenz (2015, p.66) refieren que:

Durante los primeros años de vida resulta prácticamente imposible separar el desarrollo afectivo y el desarrollo social, pues ambos van de la mano y, en gran medida, son los responsables de la adecuada evolución en el resto de ámbitos del desarrollo infantil.

Según estos autores, es necesario identificar el estilo parental según su composición y convivencia para vincular el grado de estabilidad emocional de los padres y la incidencia que tienen sobre la inteligencia emocional de los hijos. Gómez (2017) señala que dadas las condiciones que anteceden, cualquier influjo que tenga efecto sobre el sujeto que se educa, independiente de lo que acontezca y más allá de la intención de educar la emoción dentro del aula de clase, pues la emoción acontece en todos los espacios vitales.

Bandura y Walters (1974) explican que frecuentemente se encuentra la conducta imitativa y tiene en sí misma consecuencias gratificantes en la medida que el modelo exhiba una conducta socialmente efectiva; por ello la mayoría de los niños reproducen respuestas de sucesivos modelos. Esta conducta socialmente efectiva está relacionada a la narración de cuentos, canciones, fotos, otros, que permiten que los padres puedan estimular valores y orienten en la toma de decisiones; sin embargo, las actuaciones de los padres son un criterio que debe ser analizado por toda la entrega emocional que implica. Paguay y Espinoza (2014, p. 22) destaca: "El contexto familiar es importante en la personalidad, y se desarrolla a raíz del proceso de socialización, en la que el niño asimila las actitudes, valores y costumbres de la sociedad". Todo esto se compara a lo largo de los planteamientos hechos y es transcendental que los niños mantengan con los adultos comunicación continua y puedan así 
expresar sus emociones y experiencias adquiridas a lo largo de los días. En este marco para determinar el accionar de los padres en el desarrollo emocional de los hijos, se lo representa en el siguiente esquema:

Figura 1. Rol de los padres en el desarrollo emocional de los hijos

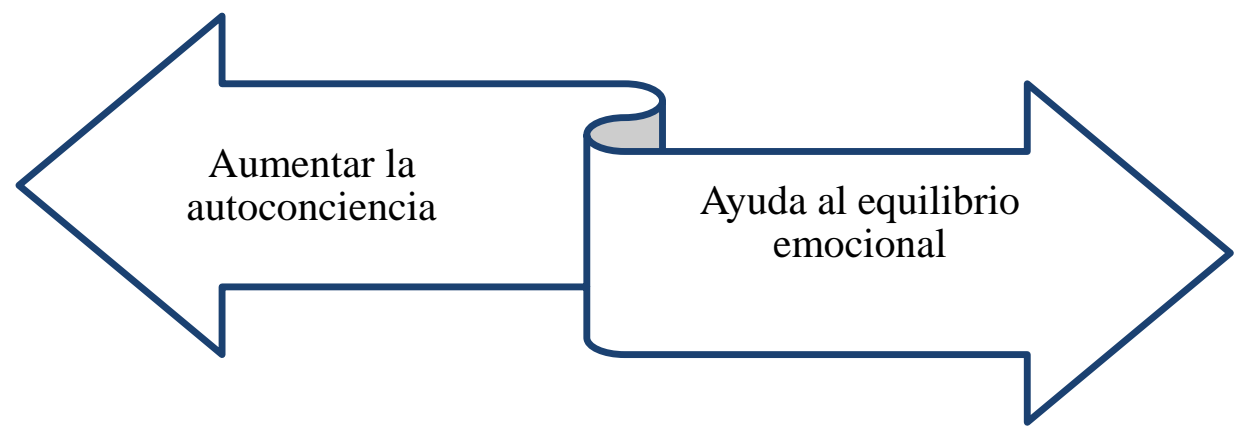

Fuente: Elaboración por las autoras

Tal como se observa, cuando los padres atienden el componente emocional, empatizan con ellos y en un primer momento van a responder con imitación y aunque hay emociones como el susto que tienen en un primer momento mayor dificultad de expresarla; sin embargo, se aprovecha en el juego para comprender las emociones de sus pares.

\section{Metodología}

Para el trabajo, se consideró a la población de un Centro de Desarrollo Infantil del Cantón Durán de la provincia del Guayas y se trabajó con el nivel inicial I que son 19 niños. El tipo de la muestra seleccionada para la investigación es no probabilístico basada en los sujetos disponibles, Este método, también conocido como muestra de conveniencia, fue seleccionada debido a que muchos de los padres por falta de tiempo no podían participar en las actividades expuestas.

La presente investigación es con un enfoque mixto y de diseño de exploratorio y descriptivo para observar y poder describir el análisis emocional para proceder luego a la recolección de datos y poder procesar la información. El registro de observación se lo planificó en cuatro meses y para ello se programó encuentros con los padres en los ambientes de aprendizaje, hora de ingreso, salida y reuniones de padres. Todos estos datos permitieron cotejar la información con los siguientes espacios:

1. Trabajo emociones con el canto. El docente del nivel de tres años tiene en su itinerario de trabajo el canto como expresión de saludo, despedida y cada que lo tenga 
planificado para propiciar un aprendizaje significativo. Esta forma de comunicarse reflejó el estado emocional cuando la persona canta y provoca emociones diferentes que van en dependencia del significado personal y la carga emocional que está implícita.

2) Trabajo emociones con el cuento: Con cuentos clásicos, infantiles, e incluso creados por los niños se dio rienda suelta a la imaginación y es una herramienta perfecta para investigar las emociones y los protagonistas de los diferentes momentos tienen a la familia como los tesoros más valiosos en la vida.

3) Trabajo emociones con cartillas: El diseño de cartillas de diferentes elementos son un componente que comunica la inteligencia emocional con actividades prácticas.

4) Espacios de convivencia para trabajo de emociones: La necesidad de expresar las emociones y sentimientos permitió que este cuarto momento recogiera todos los anteriores y los niños aprendieron en todo momento lo que hace más enriquecedor el encuentro porque todo el sentir de los niños era querer estar con sus padres y en esa dinámica familiar se cantó, contaron cuentos de historias únicas cuando sólo las miradas de los hijos con los padres ponen de manifiesto un canto de amor y al mostrar una lámina era asociada con las historias familiares.

Es importante resaltar que el niño reconoce con más facilidad la emoción que transmiten sus padres por ser objeto de imitación y es el indicador de mayor aprendizaje y adoptan las diferentes actitudes que toman los padres; por eso, ellos deben ser conscientes de las miradas de sus hijos y ser cuidadosos con todo lo que transmite. En ocasiones las tristezas que se observan en el niño, son el reflejo de lo que recibió de sus padres que se encontraban tensos o irritados. Goleman (2013, p. 14) refiere que: (...) en toda emoción hay implícita una tendencia a la acción. Basta con observar a los niños o los animales para darnos cuenta que las emociones conducen a la acción (...)”.

Esta anotación merece especial cuidado por parte del adulto al ser un modelo permanente de aprendizaje y los padres son los primeros ejemplos que reciben para seguirlos en los vínculos que van construyendo

Gráfico 2

Resultados del manejo de reconocimiento de las emociones 


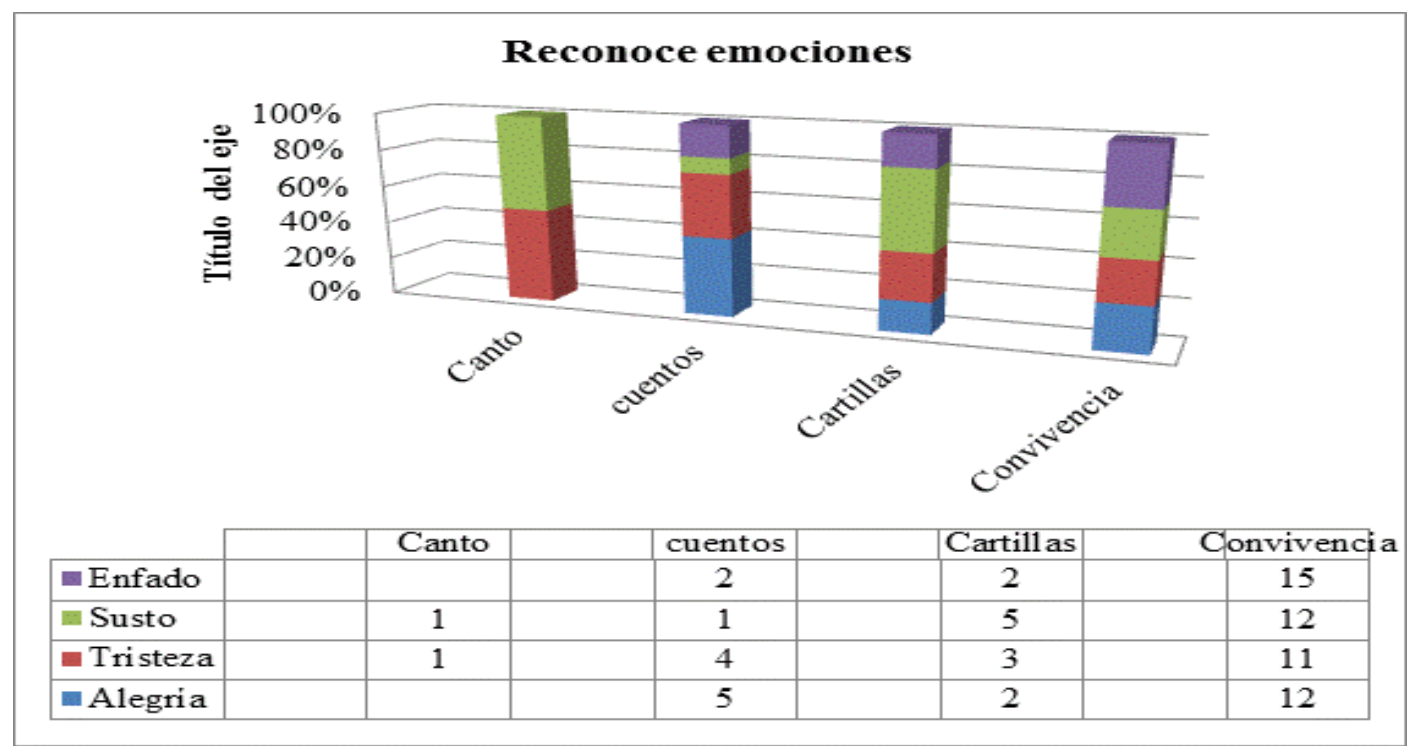

Fuente: Elaboración de las autoras

\section{Resultados}

Tabla 1

Comunicación con su hijo

\begin{tabular}{cccc}
\hline $\mathrm{N}^{\mathrm{o}}$ & Criterios & Frecuencia & Porcentaje \\
1. & Excelente & 5 & $26 \%$ \\
2. & Buena & 7 & $37 \%$ \\
3. & Regular & 7 & $37 \%$ \\
& & 19 & $100 \%$ \\
\hline
\end{tabular}

Fuente: Padres de Familia del CEI. En Durán.2017. Elaboración por las autoras

El $26 \%$ de la población, define la comunión con sus hijos con el criterio de excelente en relación al $37 \%$ que lo consideran como buena y regular. Esto muestra que los padres están conscientes que deben mejorar y prestar atención a los agentes externos que inciden en sus relaciones como por ejemplo: horarios de trabajo que acortan el tiempo de comunicación entre padre e hijos.

Tabla 2

Participación de padres en las actividades escolares de los niños

\begin{tabular}{cccc}
\hline $\mathbf{N}^{\mathbf{0}}$ & Detalle & Frecuencia & Porcentaje \\
1. & Siempre & 6 & $32 \%$ \\
\hline
\end{tabular}




\begin{tabular}{cccc}
\hline 2. & A veces & 13 & $68 \%$ \\
3. & Nunca & 0 & $0 \%$ \\
& & 19 & $100 \%$ \\
\hline
\end{tabular}

Fuente: Padres de Familia del CEI. En Durán .2017. Elaboración por las autoras

Análisis: Considerar el 68\% de los padres que responden que no pueden participar en las actividades en relación al $32 \%$, muestra un problema que llama a la reflexión de la comunidad educativa, pero acentúa un llamado urgente a los padres de familia, para ordenar las responsabilidades que debe atender y sus prioridades en el orden de la vida como padres.

En consonancia con lo anterior, los padres de familia que estén más inmersos en los movimientos de sus hijos, mayor será el control y la seguridad que le brindará a su hijo en cada labor que le toque realizar.

Tabla 3

Momentos de recreación con sus hijos

$\begin{array}{llll}\mathbf{N}^{0} & \text { Detalle } & \text { Frecuencia } & \text { Porcentaje } \\ \text { 1. } & \text { Siempre } & 9 & 47 \% \\ \text { 2. } & \text { A veces } & 10 & 53 \% \\ \text { 3. } & \text { Nunca } & 0 & 0 \%\end{array}$

Fuente: Padres de Familia del CEI. Padre Juan de Velasco.2017. Elaboración por las autoras

Análisis: Como resultado se obtuvo que el 68\% de los padres de familia encuestados señalan que a veces intervienen en las actividades y un $32 \%$ que siempre están participando en las actividades de los menores.

En la formación de los niños, es muy importante la participación de toda la comunidad educativa, pero de manera fundamental, los padres de familia tienen una responsabilidad indelegable en las actividades cotidianas de sus hijos y conforme ellos se involucren en las actividades de sus hijos, mayor será el control y la seguridad que le brindara a su hijo para que este mantenga la confianza de realizar cualquier actividad por sí solo.

El rol de los padres, debe poner énfasis en la formación de sus hijos para formar hombres y mujeres comprometidos por un verdadero cambio en una sociedad y deberá ser reforzada luego en las aulas, ya que es el segundo ambiente donde los niños se desarrollan 
con más frecuencia, es ahí donde comienza su convivencia con personas fuera de su hogar, experimentan una serie de emociones no solo propias sino también de las personas que los rodea. Deben de aceptar y comprender que todas las personas manejamos emociones ya sean de forma voluntaria o involuntaria.

Los docentes, tienen una sucesión de funciones orientadoras, una de las principales es planificar y efectuar actividades que estimulen el desarrollo integral de sus estudiantes, sin concentrarse solo en el ámbito intelectual, sino también así como ofrecer espacios para que los niños puedan auto conocerse y forjar sus propias bases de su personalidad. Se concibe entonces, como el docente recibe un papel protagónico en la educación integral de sus estudiantes, debido a que posee la habilidad de poder moldear, enseñar y entrenar a estos en habilidades que serán importantes para el resto de su vida en la convivencia con la sociedad que los rodea. Pues se convierten en figuras significativas para los niños, conservando siempre las competencias emocionales que se deben fomentar e incrementar, no solo dentro del centro de educación sino también en la sociedad.

\section{Conclusiones}

A partir de los referentes analizados con anterioridad, las autoras plantean la idea a defender que a la luz de la investigación señalan que:

- Los padres ejercen una influencia poderosa en la vida emocional de los hijos, ya que se gestionan los primeros vínculos afectivos y sociales, los mismos que servirán en la relación personal y con sus pares.

- Las expectativas de las funciones de cada miembro son muy altas y deben estar en la sintonía con los valores para que puedan transmitir a los hijos los niveles de seguridad y ellos puedan testificar el valor de la comunicación empática.

- Uno de los pilares primordiales para optimizar el comportamiento emocional es la motivación, y es uno de los métodos más convenientes para evaluar el potencial que tienen los niños para aplicarlos en la convivencia.

\section{Recomendaciones}


- Los padres de familia deben esforzarse para que proporcionen a sus hijos una educación de calidad. El comportamiento emocional, como cualquier otro perfil de la enseñanza, debe comenzar desde el hogar, y ser perfeccionado con el trabajo en aula de clases con el docente y asumiendo el compromiso de capacitarse para así poder desarrollar habilidades en el niño que le permita manejar correctamente sus emociones y así mantener una sana convivencia.

\section{Referencias}

Balabarca, I. (2018). Contexto y propuesta del radio de acción de la familia. Revista de Investigación Apuntes Universitarios, 8(2), 1-8.

Bandura, A. y Walters, R. (1974). Aprendizaje Social y Desarrollo de la Personalidad. Alianza

Editorial.

Obtenido

de http://www.soyanalistaconductual.org/aprendizaje_social_desarrollo_de_la_persona liad_albert_bandura_richard_h_walters.pdf

Goleman, D. (s.f.). Inteligencia Emocional. Kairos. Obtenido de file:///C:/Users/kguzmanh/Downloads/Goleman_Inteligencia_Emocional_Format_ Aceptable.pdf

Gómez, L. . (2017). Primera infancia y educación emocional. Revista virtual. Universidad Católica del Norte(52), 174-184. Obtenido de file://C:/Users/kguzmanh/Downloads/950-4054-2-PB.pdf

Ibarrola, B. (2014). La educación emocional en la etapa de 0 a 3 años . Recuperado el 2018, de http://www.fundaciomaresme.cat/wp-content/uploads/2014/07/21aPON\%C3\%88NCIA-2.pdf

Mancero, M. (2013). Hablar de niñez no es hablar de pequeñeces. Revista del Ministerio de Inclusión Económica y social, 15, 16.

Márquez, M. y Gaeta, M. (2017). Desarrollo de competencias emocionales en preadolescentes: el papel de padres y docentes. Revista Electrónica Interuniversitaria de Formación del Profesorado, 20(2), 221-235.

Matamoros, M. (2015). Importancia de la familia en la formación de los valores en niños de cuatro a cinco año. Sugerencias para las familias. Obtenido de (Tesis de pregrado previa a laobtención del título de licenciada en Educación Inicial. Pontificia Universidad Católica del Ecuador ): http://repositorio.puce.edu.ec/bitstream/handle/22000/8537/IMPORTANCIA\%20D E\%20LA\%20FAMILIA\%20EN\%20LA\%20FORMACI\%C3\%93N\%20DE\%20VA 
LORES\%20EN\%20NI\%C3\%91OS\%20DE\%204\%20A\%205\%20A\%C3\%91OS.\% 20\%20SUGERENCIAS\%20PARA\%20FAMILIAS.pdf?sequence=1\&isAllowed=y

Melgosa, J. (2017). Preservando la salud mental. Revista de Investigación Apuntes Universitarios, 7(2), 84-89.

Paguay, R. y Espinoza, M. (2014). El ambiente familiar y su influencia en la seguridad emocional y afectiva. Obtenido de (Tesis de pregrado para la obtención del título de licenciada en Ciencias de la Educación. Universidad Estatal de Milagro): http://repositorio.unemi.edu.ec/bitstream/123456789/2404/1/EL\%20AMBIENTE\% 20FAMILIAR\%20Y\%20SU\%20INFLUENCIA\%20EN\%20LA\%20SEGURIDAD \%20EMOCIONAL\%20Y\%20AFECTIVA..pdf

Ramírez, A. ; Ferrando, M. y Sainz A. (2015). ¡Influyen los estilos parentales y la inteligencia emocional de los padres en el desarrollo emocional de los hijos escolarizados en $2^{\circ}$ ciclo de educación infantil? Revista Acción Psicológica, 12(1), 65-78. Obtenido de https://www.redalyc.org/pdf/3440/344041426007.pdf

Soler, J., Aparicio, L, , Díaz,O., Escolano,E., \& Rodríguez, A. (2016). Inteligencia Emocional y Bienestar II. Universidad San Jorge. Recuperado el 2018, de file://C:/Users/kguzmanh/Downloads/Dialnet-InteligenciaEmocionalYBienestarII655308.pdf

Suárez, P. y Vélez, M. (2018). El papel de la familia en el desarrollo social del niño:una mirada desde la afectividad, la comunicaciónfamiliar y estilos de educación parental. PSICOESPACIOS, 12(20), 173-198. Obtenido de http://revistas.iue.edu.co/index.php/Psicoespacios/article/view/1046

UNICEF. (2012). Desarrollo emocional.Clave para la primera infancia (Primera edición ed.). Argentina: Fundación Kaleidos. Obtenido de https://www.unicef.org/ecuador/english/Desarrollo_emocional_0a3_simples.pdf 